\title{
Construction of Fuzzy Measures over Product Spaces
}

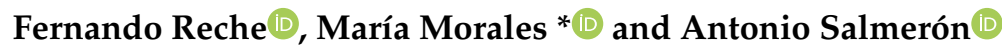 \\ Department of Mathematics and Center for the Development and Transfer of Mathematical Research to Industry \\ (CDTIME), University of Almería, 04120 Almería, Spain; fernando.reche@ual.es (F.R.); \\ antonio.salmeron@ual.es (A.S.) \\ * Correspondence: maria.morales@ual.es
}

Received: 26 August 2020; Accepted: 15 September 2020; Published: 17 September 2020

\begin{abstract}
In this paper, we study the problem of constructing a fuzzy measure over a product space when fuzzy measures over the marginal spaces are available. We propose a definition of independence of fuzzy measures and introduce different ways of constructing product measures, analyzing their properties. We derive bounds for the measure on the product space and show that it is possible to construct a single product measure when the marginal measures are capacities of order 2. We also study the combination of real functions over the marginal spaces in order to produce a joint function over the product space, compatible with the concept of marginalization, paving the way for the definition of statistical indices based on fuzzy measures.
\end{abstract}

Keywords: fuzzy measures; monotone measures; product spaces

\section{Introduction}

Fuzzy measures, also known as capacities [1], non-additive measures, or monotone measures [2] emerged as an extension of classic probabilistic measure theory by relaxing the additivity property. Fuzzy measures started to receive significant interest from the scientific community due to Choquet's work on capacities [1], but it was Sugeno [3] who first used the term fuzzy measure in relation to non-additive measures on finite domains.

Examples of fuzzy measures can also be found in contexts related to probability theory, dating back to the works by Dempster [4] and Shafer [5], who studied the substitution of the additivity property by superadditivity and subadditivity, resulting in the so-called belief and plausibility measures, respectively, and showed that they can be regarded as probability intervals.

In this paper, we are interested in the problem of constructing a fuzzy measure over a product space when fuzzy measures over the marginal spaces are available. From a practical point of view, this can be regarded as the extension of particular information, given by the fuzzy measures on the marginal spaces, to a more general setting, determined by the product space. We also study the combination of real functions defined on the marginal spaces, in order to obtain a function over the product space coherent with the initial functions.

The problem of composing fuzzy measures has received remarkable attention in the last years, but most of the works consider measures defined over the same space, and typically over the same $\sigma$-algebra [6]. With the motivation of reducing the number of parameters involved in the definition of a fuzzy measure, a variety of particular types of fuzzy measures have been studied, like $m$-separable fuzzy measures [7], that take advantage of the structure of the space where the measure is defined in order to obtain a compact representation. $k$-maxitive fuzzy measures [8] have been recently proposed as a way of encoding the interactions between the subsets of the reference set. Previously, the internal structure of fuzzy measures showing partial additivity was studied in [9]. The combination of the elements in the decomposition is carried out using different aggregation measures, including copulas [10]. 
The problem of combining fuzzy measures from marginal spaces in order to obtain a fuzzy measure over a product space has been approached from different perspectives, fundamentally based on the concept of conditioning [11-18]. Recently, the problem has been studied within the context of game theory as a way of representing coalitions between agents [19]. The measure over the product space is obtained by combining a fuzzy measure over a given $\sigma$-algebra $\mathcal{A}$ with a Lebesgue measure over the Borel $\sigma$-algebra on the interval $[0,1]$.

In this paper, we consider a more general setting, in which the measures to be combined are general fuzzy measures over potentially different spaces. The rest of the paper is organized as follows. Section 2 is devoted to give the necessary basic definitions and preliminaries. The original contributions in this paper are presented in Sections 3 and 4, covering, respectively, the combination of fuzzy measures and real functions. The paper ends with conclusions in Section 5.

\section{Preliminaries}

Definition 1. Let $X$ be a set and $\mathcal{A}$ be a non-empty class of subsets of $X$ so that $X \subset \mathcal{A}$ and $\varnothing \subset \mathcal{A}$. We say that $\mu: \mathcal{A} \longrightarrow[0,1]$ is a fuzzy measure if the following conditions hold.

1. $\mu(\varnothing)=0$.

2. $\mu(X)=1$.

3. $\forall A, B \in \mathcal{A}$ such that $A \subseteq B$ it holds that $\mu(A) \leq \mu(B)$.

4. If $\left\{A_{n}\right\}_{n \in \mathbb{N}} \in \mathcal{A}$ such that $A_{1} \subseteq A_{2} \subseteq \ldots$ and $\bigcup_{n=1}^{\infty} A_{n} \in \mathcal{A}$, then

$$
\lim _{n} \mu\left(A_{n}\right)=\mu\left(\bigcup_{n=1}^{\infty} A_{n}\right) .
$$

5. If $\left\{A_{n}\right\}_{n \in \mathbb{N}} \in \mathcal{A}$ such that $A_{1} \supseteq A_{2} \supseteq \ldots$ and $\bigcap_{n=1}^{\infty} A_{n} \in \mathcal{A}$, then

$$
\lim _{n} \mu\left(A_{n}\right)=\mu\left(\bigcap_{n=1}^{\infty} A_{n}\right) .
$$

The triplet $(X, \mathcal{A}, \mu)$ is a measurable space, and $X$ is called the reference set.

In this paper, we will only consider finite spaces that are sufficient to cover a wide range of applications domains [20]. Note that, in this case, the continuity conditions in Equations (1) and (2) always hold. Furthermore, we will also assume that $\mathcal{A}$ is the power set of $X$, i.e., the set of all subsets of $X$. In order to simplify the notation, from now on we will write $\mu_{i}$ for $\mu\left(\left\{x_{i}\right\}\right)$, and $\mu_{A}$ for $\mu(A)$.

It can be shown [21] that a fuzzy measure over a reference set of cardinality $n$ is equivalent to $n$ ! probability functions, each one of them associated with one possible permutation of the elements in the reference set. We will denote by $X^{\sigma}$ the ordering of the elements of $X$ according to permutation $\sigma$, so that $X^{\sigma}=\left\{x_{(1)}, \ldots, x_{(n)}\right\}$.

Definition 2. [21] Let $(X, \mathcal{A}, \mu)$ be a measurable space. The probability functions associated with $\mu$ and $X^{\sigma}$ are defined as the set $P_{\sigma}=\left\{p_{\sigma}\left(x_{(1)}\right), \ldots, p_{\sigma}\left(x_{(n)}\right)\right\}$ such that

$$
p_{\sigma}\left(x_{(i)}\right)= \begin{cases}\mu\left(A_{(i)}\right)-\mu\left(A_{(i+1)}\right) & \text { if } i<n, \\ \mu\left(x_{(n)}\right) & \text { if } i=n,\end{cases}
$$

where $A_{(i)}=\left\{x_{(i)}, \ldots, x_{(n)}\right\}$.

It is straightforward to show that $0 \leq p_{\sigma}\left(x_{i}\right) \leq 1$ and $\sum_{i=1}^{n} p_{\sigma}\left(x_{i}\right)=1$ for any $\sigma$. 
Definition 3. [21] Let $(X, \mathcal{A}, \mu)$ be a measurable space and let $P_{\sigma}$ be the probability function associated with $\mu$ and $X^{\sigma}$. The probability measure generated by $\mu$ and $X^{\sigma}$ is

$$
P_{\sigma}(A)=\sum_{x \in A} p_{\sigma}(x)
$$

When it is clear from the context, we will use $P_{\sigma}$ for both the probability function and probability measure.

Note that there are as many probability measures generated by $\mu$ as there are possible permutations of the element of the reference set. However, not all those measures are necessarily different.

An important property of the generated probability measure is that it bounds the underlying fuzzy measure in the extreme cases, as stated in the following proposition [22].

Proposition 1. Let $(X, \mathcal{A}, \mu)$ be a measurable space and let $\left\{P_{\sigma}\right\}_{\sigma \in S_{n}}$ be the set of all the probability measures generated by $\mu$. Then,

$$
\min _{\sigma} P_{\sigma}(A) \leq \mu(A) \leq \max _{\sigma} P_{\sigma}(A)
$$

Two types of fuzzy measures that are specially relevant for this paper are belief functions $[4,5]$ and capacities of order $2[1,23]$.

Definition 4. Let $(X, \mathcal{A}, \mu)$ be a measurable space. Given a function $m: \mathcal{P}(X) \rightarrow[0,1]$ such that $m(\varnothing)=0$ and $\sum_{A \in \mathcal{P}(X)} m(A)=1$, we say that $\mu$ is a belief function if

$$
\mu(A)=\sum_{B \in \mathcal{P}(X) \mid B \subseteq A} m(B), \quad \forall A \in \mathcal{P}(X),
$$

where $\mathcal{P}(X)$ denotes the power set of $X$.

From now on, if $\mu$ is a belief function we will denote $\mu(A)$ as $\operatorname{Bel}(A)$.

Definition 5. Let $(X, \mathcal{A}, \mu)$ be a measurable space. We say that $\mu$ is a monotone capacity of order 2 if

$$
\mu(A \cup B)+\mu(A \cap B) \geq \mu(A)+\mu(B), \quad \forall A, B \in \mathcal{A} .
$$

Definition 6. Let $(X, \mathcal{A}, \mu)$ be a measurable space. We say that $\mu$ is an alternating capacity of order 2 if

$$
\mu(A \cup B)+\mu(A \cap B) \leq \mu(A)+\mu(B), \quad \forall A, B \in \mathcal{A} .
$$

The following result [22] links capacities of order 2 with the probability measures generated by the fuzzy measure and will be key in the proof of one of the results in this paper.

Theorem 1. A fuzzy measure $\mu$ is a monotone (alternating) capacity of order 2 if and only if

$$
\mu(A)=\min _{\sigma} P_{\sigma}(A) \quad\left(\mu(A)=\max _{\sigma} P_{\sigma}(A)\right)
$$

for all $A \in \mathcal{A}$, being $\left\{P_{\sigma}\right\}_{\sigma \in S_{n}}$ the set of probability measures generated by $\mu$ (see Definition 3).

We will consider two possible scenarios related to a given product space $X_{1} \times X_{2}$, where $\times$ denotes the Cartesian product:

- A fuzzy measure defined over the product space is available. 
- Two fuzzy measures, respectively, defined over $X_{1}$ and $X_{2}$ are available, but no measure over $X_{1} \times X_{2}$ is known.

In the first scenario, we are interested in particularizing the information contained in the fuzzy measure over the unidimensional spaces. Therefore, we need to define a marginalization operation over the measure on the product space.

In the second scenario, we focus on building a fuzzy measure over the product space, by combining the two measures over the marginal spaces. Thus, we need to define an appropriate way of combining fuzzy measures.

Likewise marginal spaces, in a measurable product space we will assume the product class $\mathcal{A}_{X_{1} \times X_{2}}$ to be the power set of $X_{1} \times X_{2}$, i.e., $\mathcal{A}_{X_{1} \times X_{2}}=\mathcal{P}\left(X_{1} \times X_{2}\right)$, which is not the same as $\mathcal{P}\left(X_{1}\right) \times \mathcal{P}\left(X_{2}\right)$.

Among the possible elements of a product class, we are particularly interested in those that can be obtained from sets in the marginal space. They are called rectangles and are formally defined as follows.

Definition 7. Let $\left(X_{1}, \mathcal{A}_{X_{1}}\right)$ and $\left(X_{2}, \mathcal{A}_{X_{2}}\right)$ be two spaces where $\mathcal{A}_{X_{1}}$ and $\mathcal{A}_{X_{2}}$ are classes defined on $X_{1}$ and $X_{2}$, respectively. We define the class of rectangles of $\mathcal{A}_{X_{1} \times X_{2}}$ as

$$
\mathcal{R}=\left\{H \in \mathcal{A}_{X_{1} \times X_{2}} \mid H=A \times B \text {, where } A \in \mathcal{A}_{X_{1}}, B \in \mathcal{A}_{X_{2}}\right\} .
$$

Taking into account that we are assuming $\mathcal{A}_{X_{1} \times X_{2}}=\mathcal{P}\left(X_{1} \times X_{2}\right)$, it is easy to show that $\mathcal{R}$ is closed for intersections, but not for unions.

We will make use of the concept of triangular norm and conorm. Both are operators that raised within the context of probabilistic metric spaces [24]. They have also been widely used by the theory of fuzzy sets [25-29] as an extension of classic operations over sets.

Definition 8 ([24]). An operator $T:[0,1]^{2} \longrightarrow[0,1]$ is a triangular norm or $t$-norm for short, if it satisfies the following conditions.

1. $T(0, a)=0, T(a, 1)=a$ for all $a \in[0,1]$. (Boundary conditions)

2. $T(a, b)=T(b, a)$. (Commutativity)

3. If $a \leq c$ and $b \leq d$, then $T(a, b) \leq T(c, d)$. (Monotonicity)

4. $T(T(a, b), c)=T(a, T(b, c))$. (Asocciativity)

Example 1. Some examples of t-norms are

1. $T_{0}\left(x_{1}, x_{2}\right)= \begin{cases}\min \left\{x_{1}, x_{2}\right\} & \text { if } \max \left\{x_{1}, x_{2}\right\}=1, \\ 0 & \text { otherwise. }\end{cases}$

2. $T_{1}\left(x_{1}, x_{2}\right)=\max \left\{x_{1}+x_{2}-1,0\right\}$.

3. $T_{2}\left(x_{1}, x_{2}\right)=x_{1} x_{2}$.

4. $T_{3}\left(x_{1}, x_{2}\right)=\min \left\{x_{1}, x_{2}\right\}$.

Note that any $t$-norm $T$ is always bounded by $T_{0}$ and $T_{3}$ in the following way.

$$
T_{0}\left(x_{1}, x_{2}\right) \leq T\left(x_{1}, x_{2}\right) \leq T_{3}\left(x_{1}, x_{2}\right) .
$$

Definition 9 ([24]). An operator $T:[0,1]^{2} \longrightarrow[0,1]$ is a triangular conorm or $t$-conorm for short, if it satisfies the following properties.

1. $S(1, a)=1, S(a, 0)=a$ for all $a \in[0,1]$. (Boundary conditions)

2. $S(a, b)=S(b, a)$. (Commutativity)

3. If $a \leq c$ and $b \leq d$, then $S(a, b) \leq S(c, d)$. (Monotonicity)

4. $\quad S(S(a, b), c)=S(a, S(b, c))$. (Asocciativity) 
Given any t-norm $T$, a t-conorm $S$ can always be constructed as

$$
S(a, b)=1-T(1-a, 1-b)
$$

Example 2. Applying Equation (12) to the t-norms in Example 1, we obtain the following t-conorms.

1. $S_{0}\left(x_{1}, x_{2}\right)= \begin{cases}\max \left\{x_{1}, x_{2}\right\} & \text { if } \min \left\{x_{1}, x_{2}\right\}=0, \\ 1 & \text { otherwise. }\end{cases}$

2. $S_{1}\left(x_{1}, x_{2}\right)=\min \left\{x_{1}+x_{2}, 1\right\}$.

3. $S_{2}\left(x_{1}, x_{2}\right)=x_{1}+x_{2}-x_{1} x_{2}$.

4. $S_{3}\left(x_{1}, x_{2}\right)=\max \left\{x_{1}, x_{2}\right\}$.

A similar boundary condition as expressed in Equation (11) for t-norms, holds for t-conorms:

$$
S_{3}\left(x_{1}, x_{2}\right) \leq S\left(x_{1}, x_{2}\right) \leq S_{0}\left(x_{1}, x_{2}\right)
$$

A thorough study of the use of $t$-norms and $t$-conorms in the context of fuzzy measures and fuzzy sets can be found in [28].

Functions can be integrated with respect to a fuzzy measure using Choquet integral, which is a generalization of Lebesgue integral to non-additive monotone measures [1]. In the particular case of additive measures, Choquet and Lebesgue integrals coincide. It is formally defined as follows.

Definition 10. Let $(X, \mathcal{A}, \mu)$ be a measurable space, and let $h$ be a measurable real function of $X$. The Choquet integral of $h$ with respect to $\mu$ is

$$
\oint_{A} h \circ \mu=\int_{-\infty}^{0}\left(\mu\left(H_{\alpha} \cap A\right)-1\right) d \alpha+\int_{0}^{\infty} \mu\left(H_{\alpha} \cap A\right) d \alpha
$$

where $A \in \mathcal{A}$ and $H_{\alpha}$ are the $\alpha$-cuts of $h$, defined as

$$
H_{\alpha}=\{x \in X / h(x) \geq \alpha\}
$$

If the reference set is finite, the integral can be expressed as

$$
\oint h \circ \mu=h\left(x_{(1)}\right) \mu\left(A_{(1)}\right)+\sum_{i=2}^{n} \mu\left(A_{(i)}\right)\left[h\left(x_{(i)}\right)-h\left(x_{(i-1)}\right)\right]
$$

where $X^{\sigma}$ is an ordering such that $h\left(x_{(1)}\right) \leq h\left(x_{(2)}\right) \leq \ldots \leq h\left(x_{(n)}\right)$ and the sets $A_{(i)}$ are of the form $\left\{x_{(i)}, x_{(i+1)}, \ldots, x_{(n)}\right\}$.

\section{Combining Fuzzy Measures}

The main difficulty when combining fuzzy measures from marginal spaces in order to obtain a fuzzy measure over a product space is that, unlike probability measures, we cannot follow a procedure based on extending the measures, as additivity is required [11-18].

For instance, for sets of the form $A \times B$, where $A \in \mathcal{A}_{X_{1}}$ and $B \in \mathcal{A}_{X_{2}}$, we could define $\mu(A \times B)=$ $\mu_{X_{1}}(A) \otimes \mu_{X_{2}}(B)$ for some appropriate operator $\otimes$. In the case of probability measures, this would suffice as, due to additivity, the measure can easily be extended to arbitrary sets of $X_{1} \times X_{2}$ using integrals [30]. More precisely, in the case of additive measures, the product measure for sets of the form $A \times B$, is given by $\mu_{X_{1}}(A) \mu_{X_{2}}(B)$, while for the rest of sets $Q \subseteq X_{1} \times X_{2}$, the product measure is computed as 


$$
\mu(Q)=\int \mu_{X_{2}}\left(Q_{x_{1}}\right) \mu_{X_{1}}\left(d x_{1}\right)=\int \mu_{X_{1}}\left(Q_{x_{2}}\right) \mu_{X_{2}}\left(d x_{2}\right)
$$

where $Q_{x_{1}}=\left\{x_{2} \in X_{2} \mid\left(x_{1}, x_{2}\right) \in Q\right\}$ and $Q_{x_{2}}=\left\{x_{1} \in X_{1} \mid\left(x_{1}, x_{2}\right) \in Q\right\}$.

The same construction is not always possible In the case of non-additive measures, because the integrals in Equation (17) can be different [14]. It happens, for instance, if we use Choquet integral [1].

\section{1. $\odot-I n d e p e n d e n t ~ M e a s u r e s$}

Consider two measurable spaces $\left(X_{1}, \mathcal{A}_{X_{1}}, \mu_{1}\right)$ and $\left(X_{2}, \mathcal{A}_{X_{2}}, \mu_{2}\right)$. Our goal is to construct a fuzzy measure over the product space in a sensible way. We start off by defining a fuzzy measure over the product space, compatible with the marginal measures:

Definition 11. A product fuzzy measure of $\mu_{1}$ and $\mu_{2}$ is a function $\mu_{12}: \mathcal{A}_{X_{1} \times X_{2}} \longrightarrow[0,1]$ satisfying

1. $\mu_{12}(\varnothing)=0, \mu_{12}\left(X_{1} \times X_{2}\right)=1$.

2. For all $A, B \in \mathcal{A}_{X_{1} \times X_{2}}$ such that $A \subseteq B$ it holds that $\mu_{12}(A) \leq \mu_{12}(B)$.

3. For all $A \in \mathcal{A}_{X_{1}}$, it holds that $\mu_{12}\left(A \times X_{2}\right)=\mu_{1}(A)$.

4. For all $B \in \mathcal{A}_{X_{2}}$, it holds that $\mu_{12}\left(X_{1} \times B\right)=\mu_{2}(B)$.

The next step is to guarantee that the composition of measures using the product in Definition 11 is compatible with the concept of independence. More precisely, assuming independence between two fuzzy measures, their product fuzzy measure should be possible to be obtained using exclusively the two original fuzzy measures. In this work, we will assume that two fuzzy measures are independent if they can be composed resulting in a product fuzzy measure within the class $\mathcal{R}$. This is formally defined through the concept of $\odot$-independence.

Definition 12. Let $\left(X_{1}, \mathcal{A}_{X_{1}}, \mu_{1}\right)$ and $\left(X_{2}, \mathcal{A}_{X_{2}}, \mu_{2}\right)$ be measurable spaces. We say that $\mu_{1}$ and $\mu_{2}$ are $\odot$-independent fuzzy measures if there exists a product fuzzy measure $\mu_{12}^{\odot}$ satisfying that for any $H \in \mathcal{R}$ it holds that

$$
\mu_{12}^{\odot}(H)=\mu_{1}(A) \odot \mu_{2}(B),
$$

where $H=A \times B$ and $\odot$ is a t-norm.

From now on we will refer to this measure as the $\odot$-independent product of $\mu_{1}$ and $\mu_{2}$.

The next proposition shows that the $\odot$-independent product results in a well defined fuzzy measure on $\mathcal{R}$.

Proposition 2. Let $\left(X_{1}, \mathcal{A}_{X_{1}}, \mu_{1}\right)$ and $\left(X_{2}, \mathcal{A}_{X_{2}}, \mu_{2}\right)$ be measurable spaces. The $\odot$-independent product of $\mu_{1}$ and $\mu_{2}, \mu_{12}^{\odot}$, is a fuzzy measure on $\mathcal{R}$.

Proof. Let $H \in \mathcal{R}$ such that $H=A \times B$ with $A \in \mathcal{A}_{X_{1}}$ and $B \in \mathcal{A}_{X_{2}}$. We have to show that the conditions in Definition 11 are satisfied by $\mu_{12}^{\odot}$.

1. It is clear that $\varnothing \in \mathcal{R}$ as it can be expressed as $\varnothing=A \times B$ if at least one of them $(A$ or $B)$ is the empty set. In such case, $\mu_{1}(A), \mu_{2}(B)$, or both are equal to zero (since they are fuzzy measures). Hence, $\mu_{12}^{\odot}(\varnothing)=0$ because $\odot$ is a t-norm.

2. $X_{1} \times X_{2}$ trivially belongs to $\mathcal{R}$, and thus $\mu_{12}^{\odot}\left(X_{1} \times X_{2}\right)=\mu_{1}\left(X_{1}\right) \odot \mu_{2}\left(X_{2}\right)=1 \odot 1=1$.

3. Let $H_{1} \subseteq H_{2} \in \mathcal{R}$. Assume that $H_{1}=A_{1} \times B_{1}$ and $H_{2}=A_{2} \times B_{2}$. Then, $A_{1} \subseteq A_{2}$ and $B_{1} \subseteq B_{2}$. As the t-norm $\odot$ is a monotone operator, it holds that

$$
\mu_{12}^{\odot}\left(H_{1}\right)=\mu_{1}\left(A_{1}\right) \odot \mu_{2}\left(B_{1}\right) \leq \mu_{1}\left(A_{2}\right) \odot \mu_{2}\left(B_{2}\right)=\mu_{12}^{\odot}\left(H_{2}\right) \text {, }
$$

which means that $\mu_{12}^{\odot}$ is monotone. 
4. Given $A \in \mathcal{A}_{X_{1}}$, it holds that $A \times X_{2} \in \mathcal{R}$ and therefore

$$
\mu_{12}^{\odot}\left(A \times X_{2}\right)=\mu_{1}(A) \odot \mu_{2}\left(X_{2}\right)=\mu_{1}(A) \odot 1=\mu_{1}(A) .
$$

5. Analogously, we can see that $\mu_{12}^{\odot}\left(X_{1} \times B\right)=\mu_{2}(B)$.

Example 3. Let $\left(X_{1}, \mathcal{A}_{X_{1}}, \mu_{1}\right)$ and $\left(X_{2}, \mathcal{A}_{X_{2}}, \mu_{2}\right)$ be probabilistic spaces. Therefore, $\mu_{1}$ and $\mu_{2}$ are additive measures and $\mathcal{A}_{X_{1}}$ and $\mathcal{A}_{X_{2}}$ are algebras. By letting $\odot$ be equal to the product between real numbers, denoted by $\times$, we can define the $\times$-independent product of $\mu_{1}$ and $\mu_{2}$ as

$$
\mu_{12}^{\times}(H)=\mu_{1}(A) \times \mu_{2}(B)
$$

with $H=A \times B \in \mathcal{R}$. It is known that, in this case, there exists a unique additive product measure defined over the smaller algebra generated by the corresponding rectangles [30].

Similar examples can be found for different types of fuzzy measures, as for instance, possibility measures [31,32], illustrating that, given a $\odot$-independent fuzzy measure, it is possible to find a unique product measure compatible with the initial ones. However, uniqueness does not hold in general, so we will instead pursue the idea of defining bounds where the product fuzzy measure lies.

Definition 13. Let $\left(X_{1}, \mathcal{A}_{X_{1}}, \mu_{1}\right)$ and $\left(X_{2}, \mathcal{A}_{X_{2}}, \mu_{2}\right)$ be measurable spaces. We define the $\odot$-exterior product measure for any $H \in \mathcal{A}_{X_{1} \times X_{2}}$ as

$$
\bar{\mu}_{12}^{\odot}(H)=\min _{A \times B \supseteq H} \mu_{1}(A) \odot \mu_{2}(B)
$$

where $\odot$ is a t-norm.

Definition 14. Let $\left(X_{1}, \mathcal{A}_{X_{1}}, \mu_{1}\right)$ and $\left(X_{2}, \mathcal{A}_{X_{2}}, \mu_{2}\right)$ be measurable spaces. We define the $\odot-$ interior product measure for any $H \in \mathcal{A}_{X_{1} \times X_{2}}$ as

$$
\underline{\mu}_{12}^{\odot}(H)=\max _{A \times B \subseteq H} \mu_{1}(A) \odot \mu_{2}(B)
$$

where $\odot$ is a t-norm.

These definitions are more general than the ones introduced in [13], where the product t-norm is used instead. Figure 1 shows a representation of the $\odot$-interior and exterior product measures corresponding, respectively, to the contained rectangle of larger measure and the containing rectangle of lower measure.

The next results shows that both $\odot$-interior and exterior product measures are indeed product fuzzy measures.

Proposition 3. Let $\left(X_{1}, \mathcal{A}_{X_{1}}, \mu_{1}\right)$ and $\left(X_{2}, \mathcal{A}_{X_{2}}, \mu_{2}\right)$ be measurable spaces. Then, the $\odot$-interior (resp. exterior) product measure is a product fuzzy measure of $\mu_{1}$ and $\mu_{2}$.

Proof. We will show that $\underline{\mu}_{12}^{\odot}(H)$ satifies Definition 11 . The proof for the $\odot$-exterior product measure is analogous. Note that

$$
\underline{\mu}_{12}^{\odot}(\varnothing)=\max _{A \times B \subseteq \varnothing} \mu_{1}(A) \odot \mu_{2}(B)=0,
$$


as $A \times B \subseteq \varnothing$, and therefore at least one of them will be equal to $\varnothing$. We have also used that, as $\mu_{1}$ and $\mu_{2}$ are fuzzy measures and $\odot$ is a t-norm, the value of the $\odot$-interior product measure for the empty set is zero.

The value of the $\odot$-interior product measure for $X_{1} \times X_{2}$ is

$$
\begin{aligned}
\underline{\mu}_{12}^{\odot}\left(X_{1} \times X_{2}\right) & =\max _{A \times B \subseteq X_{1} \times X_{2}} \mu_{1}(A) \odot \mu_{2}(B) \\
& =\mu_{1}\left(X_{1}\right) \odot \mu_{2}\left(X_{2}\right) \\
& =1 \odot 1=1 .
\end{aligned}
$$

Now we will show that monotonicity also holds. Let $H_{1} \subseteq H_{2} \subseteq X_{1} \times X_{2}$, then

$$
\begin{aligned}
\underline{\mu}_{12}^{\odot}\left(H_{1}\right) & =\max _{A_{1} \times B_{1} \subseteq H_{1}} \mu_{1}\left(A_{1}\right) \odot \mu_{2}\left(B_{1}\right) \\
& =\max _{A_{1} \times B_{1} \subseteq H_{1} \subseteq H_{2}} \mu_{1}\left(A_{1}\right) \odot \mu_{2}\left(B_{1}\right) \\
& \leq \max _{A_{2} \times B_{2} \subseteq H_{2}} \mu_{1}\left(A_{2}\right) \odot \mu_{2}\left(B_{2}\right) \\
& =\underline{\mu}_{12}^{\odot}\left(H_{2}\right) .
\end{aligned}
$$

That is, as $H_{1} \subseteq H_{2}$ and $\odot$ is a t-norm (and thus a monotone operator), in the worst case, the interior rectangle of larger measure in $H_{2}$ has, at least, the same measure as the one in $H_{1}$.

Let us check now the compatibility with marginalization. As $A \times X_{2}$ and $X_{1} \times B$ belong to $\mathcal{R}$, and taking into account that $\odot$ is a t-norm, it holds that

$$
\begin{aligned}
& \underline{\mu}_{12}^{\odot}\left(A \times X_{2}\right)=\mu_{1}(A) \odot \mu_{2}\left(X_{2}\right)=\mu_{1}(A), \\
& \underline{\mu}_{12}^{\odot}\left(X_{1} \times B\right)=\mu_{1}\left(X_{1}\right) \odot \mu_{2}(B)=\mu_{2}(B) .
\end{aligned}
$$

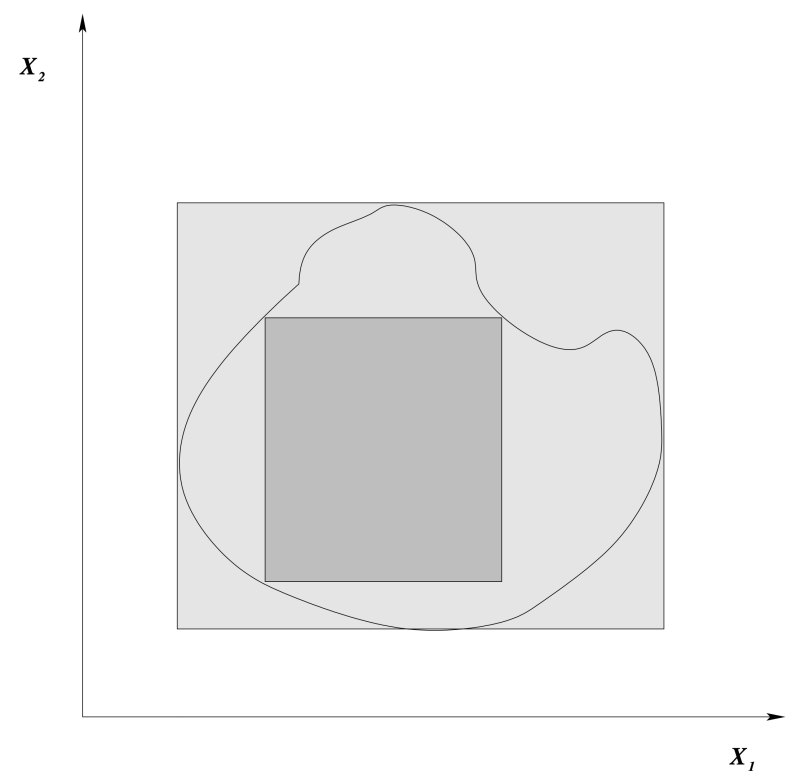

Figure 1. A representation of internal and external product measures.

Note that, for the particular case of the class $\mathcal{R}$, both measures turn out to be the same, i.e., for all $H \in \mathcal{R}$, it holds that

$$
\underline{\mu}_{12}^{\odot}(H)=\mu_{12}^{\odot}(H)=\bar{\mu}_{12}^{\odot}(H)
$$


Finally, the next result states how, in the general case, $\underline{\mu}_{12}^{\odot}$ and $\bar{\mu}_{12}^{\odot}$ conform lower and upper bounds, respectively, for any $\odot$-independent product fuzzy measure.

Proposition 4. Let $\left(X_{1}, \mathcal{A}_{X_{1}}, \mu_{1}\right)$ and $\left(X_{2}, \mathcal{A}_{X_{2}}, \mu_{2}\right)$ be measurable spaces. Given any $\odot$-independent product of $\mu_{1}$ and $\mu_{2}$, it holds that for all $C \in \mathcal{A}_{X_{1} \times X_{2}}$,

$$
\underline{\mu}_{12}^{\odot}(C) \leq \mu_{12}^{\odot}(C) \leq \bar{\mu}_{12}^{\odot}(C) .
$$

Proof. We will only develop the proof for the lower bound. The upper bound case is analogous.

As $\mu_{12}^{\odot}$ is a monotone measure, for any $A \times B \in \mathcal{R}$, with $A \times B \subseteq C \in \mathcal{A}_{X_{1} \times X_{2}}$ it holds that $\mu_{12}^{\odot}(A \times B) \leq \mu(C)$, and therefore

$$
\mu_{12}^{\odot}(C) \geq \max _{A \times B \subseteq C} \mu_{12}^{\odot}(A \times B)=\max _{A \times B \subseteq C} \mu_{1}(A) \odot \mu_{2}(B)=\underline{\mu}_{12}^{\odot}(C),
$$

taking into account that $\mu_{12}^{\odot}$ satisfies that $\mu_{12}^{\odot}(A \times B)=\mu_{1}(A) \odot \mu_{2}(B)$.

Up to this point, we have only been able to provide a way to obtain a combined fuzzy measure when working within the class $\mathcal{R}$. Outside it only bounds have been obtained. In the next section we solve this problem for some particular kinds of fuzzy measures.

\subsection{Maximin Product}

We will elaborate on an idea introduced in [17] based on an alternative representation of general fuzzy measures. Given any fuzzy measure, it is always possible to construct classes of measures bounding it. Such is the case of

$$
\mathcal{M}_{P}(\mu)=\{P \in \mathfrak{P} \mid P(A) \geq \mu(A) \forall A \in \mathcal{A}\}
$$

and

$$
\mathcal{M}_{\text {Bel }}(\mu)=\{\operatorname{Bel}(A) \in \mathfrak{B} \mid \operatorname{Bel}(A) \leq \mu(A) \forall A \in \mathcal{A}\},
$$

where $\mathfrak{P}$ denotes the set of all probability measures and $\mathfrak{B}$ is the set of all belief measures.

It can be shown $[17,18]$ that any fuzzy measure $\mu$ can be represented using elements of the classes defined above as

$$
\mu(A)=\max _{\beta \in \mathcal{M}_{B e l}(\mu)} \min _{P \in \mathcal{M}_{P}(\beta)} P(A),
$$

and the Choquet integral of any function $h$ with respect to $\mu$ can also be computed as

$$
\oint h \circ \mu=\max _{\beta \in \mathcal{M}_{B e l}(\mu)} \min _{P \in \mathcal{M}_{P}(\beta)} \int h d P .
$$

In other words, given any fuzzy measure, there is always a probability measure whose value matches it for a given subset of the reference set.

Taking this representation as a basis, we have the conditions to propose a product fuzzy measure over $X_{1} \times X_{2}$ as follows.

Definition 15. Let $\left(X_{1}, \mathcal{A}_{X_{1}}, \mu_{1}\right)$ and $\left(X_{2}, \mathcal{A}_{X_{2}}, \mu_{2}\right)$ be measurable spaces. We define the maximin product measure as

$$
\mu_{1} \otimes \mu_{2}(A)=\max _{\substack{\beta_{i} \in \mathcal{M}_{B e l}\left(\mu_{i}\right) \\ i=1,2}} \min _{\substack{P_{i} \in \mathcal{M}_{P}\left(\beta_{i}\right) \\ i=1,2}} P_{1} \otimes P_{2}(A),
$$

where $P_{1} \otimes P_{2}$ is the standard product for probability measures. 
The main drawback of this definition, from a practical point of view, is that it involves solving an optimization problem over very general classes of fuzzy measures. However, we will show that an approximation can be easily obtained taking advantage of the representation of a fuzzy measure by means of a set of probability measures. Remarkably, for some particular types of fuzzy measures, we will also show that, rather than just bounds, we can obtain a precise product fuzzy measure.

Definition 16. Let $\left(X_{1}, \mathcal{A}_{X_{1}}, \mu_{1}\right)$ and $\left(X_{2}, \mathcal{A}_{X_{2}}, \mu_{2}\right)$ be measurable spaces and $P_{\sigma_{1}}^{\mu_{1}}$ and $P_{\sigma_{2}}^{\mu_{2}}$ be the probability functions associated with $X_{1}^{\sigma_{1}}$ and $X_{2}^{\sigma_{2}}$, respectively. We define the lower product $p$-measure as

$$
\underline{\mathfrak{m}}_{12}(C)=\min _{\sigma_{1}, \sigma_{2}}\left[P_{\sigma_{1}}^{\mu_{1}} \otimes P_{\sigma_{2}}^{\mu_{2}}(C)\right],
$$

where $\otimes$ is the standard probabilistic product.

Definition 17. Given the conditions in Definition 16, we define the upper product p-measure as

$$
\overline{\mathfrak{m}}_{12}(C)=\max _{\sigma_{1}, \sigma_{2}}\left[P_{\sigma_{1}}^{\mu_{1}} \otimes P_{\sigma_{2}}^{\mu_{2}}(C)\right],
$$

where $\otimes$ is the standard probabilistic product.

The construction of these measures comprises the following steps, assuming measurable spaces $\left(X_{1}, \mathcal{A}_{X_{1}}, \mu_{1}\right)$ and $\left(X_{2}, \mathcal{A}_{X_{2}}, \mu_{2}\right)$ with cardinalities $n_{1}$ and $n_{2}$, respectively.

- Compute the $n_{1}$ ! probability measures associated with $\mu_{1}$ and the $n_{2}$ ! corresponding to $\mu_{2}$.

- Compute the $n_{1} ! \times n_{2}$ ! product probability measures using the standard product.

- The product $\mathrm{p}$-measures are computed, for any given set, using the smaller and larger product probability measure.

In fact, there is no need to know the value of the probabilities for all the possible subsets for all the permutations, as the probability measures are additive, and therefore it suffices to know the values for the elementary events (singletones).

Example 4. Consider two measurable spaces, $\left(X_{1}, \mathcal{A}_{X_{1}}, \mu_{1}\right)$ and $\left(X_{2}, \mathcal{A}_{X_{2}}, \mu_{2}\right)$, both with cardinality 3. Table 1 shows an example of a fuzzy measure with all its possible values specified, as well as the associated probability measures corresponding to each permutation of the subsets of the reference set.

As both reference sets have cardinality 3, each one of them has three proper sets (i.e., excluding the total and empty sets). Thus, $X_{1} \times X_{2}$ contains 9 elements and $2^{9}-2=510$ proper subsets. For the sake of readability, we will use the notation $\left(x_{1 i}, x_{2 j}\right)=z_{i j}$, meaning that

$$
X_{1} \times X_{2}=\left\{z_{11}, z_{12}, z_{13}, z_{21}, z_{22}, z_{23}, z_{31}, z_{32}, z_{33}\right\} .
$$

Let us see how to compute, from the data in Table 1, the product p-measures for some subsets:

- $\quad$ Consider the unitary subset $z_{11}$.

$$
\underline{\mathfrak{m}}_{12}\left(\left\{z_{11}\right\}\right)=\min _{\sigma_{1}, \sigma_{2}} P_{\sigma_{1}}^{\mu_{1}}\left(\left\{x_{11}\right\}\right) P_{\sigma_{2}}^{\mu_{2}}\left(\left\{x_{21}\right\}\right)=0.1 \cdot 0.1=0.01 .
$$

The calculation for unitary subsets is easy, as it requires just to search the permutation with lower value in both components, which in this case are $P_{(3,1,2)}^{\mu_{1}}$ and $P_{(2,3,1)}^{\mu_{2}}$. 
- Now we will consider a set from class $\mathcal{R}$, namely, $\left\{z_{22}, z_{23}\right\}$,

$$
\begin{aligned}
\underline{\mathfrak{m}}_{12}\left(\left\{z_{22}, z_{23}\right\}\right) & =\min _{\sigma_{1}, \sigma_{2}}\left[P_{\sigma_{1}}^{\mu_{1}}\left(\left\{x_{12}\right\}\right) P_{\sigma_{2}}^{\mu_{2}}\left(\left\{x_{22}\right\}\right)+P_{\sigma_{1}}^{\mu_{1}}\left(\left\{x_{12}\right\}\right) P_{\sigma_{2}}^{\mu_{2}}\left(\left\{x_{23}\right\}\right)\right] \\
& =\min _{\sigma_{1}, \sigma_{2}}\left[P_{\sigma_{1}}^{\mu_{1}}\left(\left\{x_{12}\right\}\right)\left[P_{\sigma_{2}}^{\mu_{2}}\left(\left\{x_{22}\right\}\right)+P_{\sigma_{2}}^{\mu_{2}}\left(\left\{x_{23}\right\}\right)\right]\right] \\
& =\min _{\sigma_{1}, \sigma_{2}}\left[P_{\sigma_{1}}^{\mu_{1}}\left(\left\{x_{12}\right\}\right) P_{\sigma_{2}}^{\mu_{2}}\left(\left\{x_{22}, x_{23}\right\}\right)\right] \\
& =0.2 \cdot 0.6=0.12 .
\end{aligned}
$$

The calculations here are analogous to the unitary set case, as it is enough to find the permutations returning the minimum value for the projections. In this case, they are $P_{(3,2,1)}^{\mu_{1}}$ for $\left\{x_{12}\right\}$ and $P_{(1,3,2)}^{\mu_{2}}$ for $\left\{x_{22}, x_{23}\right\}$.

- Consider now a subset outside the class $\mathcal{R}$, for instance $\left\{z_{11}, z_{22}\right\}$. We obtain

$$
\begin{aligned}
\underline{\mathfrak{m}}_{12}\left(\left\{z_{11}, z_{22}\right\}\right) & =\min _{\sigma_{1}, \sigma_{2}}\left[P_{\sigma_{1}}^{\mu_{1}}\left(\left\{x_{11}\right\}\right) P_{\sigma_{2}}^{\mu_{2}}\left(\left\{x_{21}\right\}\right)+P_{\sigma_{1}}^{\mu_{1}}\left(\left\{x_{12}\right\}\right) P_{\sigma_{2}}^{\mu_{2}}\left(\left\{x_{22}\right\}\right)\right] \\
& =0.3 \cdot 0.1+0.2 \cdot 0.6=0.15 .
\end{aligned}
$$

This case is more complicated, as it requires exploring all the possible combination of products and finding the permutation returning the lowest value. In this case, they are $P_{(3,2,1)}^{\mu_{1}}$ and $P_{(2,3,1)}^{\mu_{2}}$.

Table 1. Probability measures generated by two sample fuzzy measures.

\begin{tabular}{cccccccc}
\hline $\mathcal{P}\left(X_{1}\right)$ & $\mu_{1}$ & $P_{(1,2,3)}^{\mu_{1}}$ & $P_{(1,3,2)}^{\mu_{1}}$ & $P_{(2,1,3)}^{\mu_{1}}$ & $P_{(2,3,1)}^{\mu_{1}}$ & $P_{(3,1,2)}^{\mu_{1}}$ & $P_{(3,2,1)}^{\mu_{1}}$ \\
\hline$x_{11}$ & 0.3 & 0.2 & 0.2 & 0.15 & 0.3 & 0.1 & 0.3 \\
$x_{12}$ & 0.4 & 0.35 & 0.4 & 0.4 & 0.4 & 0.4 & 0.2 \\
$x_{13}$ & 0.45 & 0.45 & 0.4 & 0.45 & 0.3 & 0.5 & 0.5 \\
$x_{11}, x_{12}$ & 0.5 & 0.55 & 0.6 & 0.55 & 0.7 & 0.5 & 0.5 \\
$x_{11}, x_{13}$ & 0.6 & 0.65 & 0.6 & 0.6 & 0.6 & 0.6 & 0.8 \\
$x_{12}, x_{13}$ & 0.8 & 0.8 & 0.8 & 0.85 & 0.7 & 0.9 & 0.7 \\
\hline $\mathcal{P}\left(X_{2}\right)$ & $\mu_{2}$ & $P_{(1,2,3)}^{\mu_{2}}$ & $P_{(1,3,2)}^{\mu_{2}}$ & $P_{(2,1,3)}^{\mu_{2}}$ & $P_{(2,3,1)}^{\mu_{2}}$ & $P_{(3,1,2)}^{\mu_{2}}$ & $P_{(3,2,1)}^{\mu_{2}}$ \\
\hline$x_{21}$ & 0.1 & 0.4 & 0.4 & 0.2 & 0.1 & 0.2 & 0.1 \\
$x_{22}$ & 0.5 & 0.4 & 0.5 & 0.6 & 0.6 & 0.5 & 0.6 \\
$x_{23}$ & 0.2 & 0.2 & 0.1 & 0.2 & 0.3 & 0.3 & 0.3 \\
$x_{21}, x_{22}$ & 0.7 & 0.8 & 0.9 & 0.8 & 0.7 & 0.7 & 0.7 \\
$x_{21}, x_{23}$ & 0.4 & 0.6 & 0.5 & 0.4 & 0.4 & 0.5 & 0.4 \\
$x_{22}, x_{23}$ & 0.6 & 0.6 & 0.6 & 0.8 & 0.9 & 0.8 & 0.9 \\
\hline
\end{tabular}

The calculations are similar for the upper product p-measure, resulting in

$$
\begin{aligned}
\overline{\mathfrak{m}}_{12}\left(\left\{z_{11}\right\}\right) & =0.12 \\
\overline{\mathfrak{m}}_{12}\left(\left\{z_{22}, z_{23}\right\}\right) & =0.36 \\
\overline{\mathfrak{m}}_{12}\left(\left\{z_{11}, z_{22}\right\}\right) & =0.32 .
\end{aligned}
$$

Note that the product p-measures provide an interval of measure over the product space, but they are obtained in a rather different way than the exterior and interior product measures defined in Equations (20) and (21). We will analyze now some remarkable properties of product p-measures, first of all checking that they are actually fuzzy measures. 
Proposition 5. $\underline{\mathfrak{m}}_{12}$ and $\overline{\mathfrak{m}}_{12}$ are fuzzy measures.

Proof. Consider $\underline{\mathfrak{m}}_{12}(C)=\min _{\sigma_{1}, \sigma_{2}} P_{\sigma_{1}}^{\mu_{1}} \otimes P_{\sigma_{2}}^{\mu_{2}}(C)$. As $\otimes$ is the standard probabilistic product and we are assuming the reference set to be finite, it holds that there exist two permutations $\sigma_{1}$ and $\sigma_{2}$ such that

$$
\underline{\mathfrak{m}}_{12}(C)=\sum_{\left(x_{1 i}, x_{2 j}\right) \in C} p_{\sigma_{1}}\left(x_{1 i}\right) p_{\sigma_{2}}\left(x_{2 j}\right) \text {. }
$$

Given $C \subset H$, it follows from Definition 16 that there exist two permutations $\tau_{1}$ and $\tau_{2}$ such that

$$
\begin{aligned}
\underline{\mathfrak{m}}_{12}(H) & =\sum_{\left(x_{1 i}, x_{2 j}\right) \in H} p_{\tau_{1}}\left(x_{1 i}\right) p_{\tau_{2}}\left(x_{2 j}\right) \\
& =\sum_{\left(x_{1 i}, x_{2 j}\right) \in C} p_{\tau_{1}}\left(x_{1 i}\right) p_{\tau_{2}}\left(x_{2 j}\right)+\sum_{\left(x_{1 i}, x_{2 j}\right) \in H-C} p_{\tau_{1}}\left(x_{1 i}\right) p_{\tau_{2}}\left(x_{2 j}\right)
\end{aligned}
$$

As $\sigma_{1}$ and $\sigma_{2}$ are the permutations that minimize the product probability of $C$, it holds that

$$
\begin{aligned}
\underline{\mathfrak{m}}_{12}(H) & \geq \sum_{\left(x_{1 i}, x_{2 j}\right) \in C} p_{\sigma_{1}}\left(x_{1 i}\right) p_{\sigma_{2}}\left(x_{2 j}\right)+\sum_{\left(x_{1 i}, x_{2 j}\right) \in H-C} p_{\tau_{1}}\left(x_{1 i}\right) p_{\tau_{2}}\left(x_{2 j}\right) \\
& =\underline{\mathfrak{m}}_{12}(C)+\sum_{\left(x_{1 i}, x_{2 j}\right) \in H-C} p_{\tau_{1}}\left(x_{1 i}\right) p_{\tau_{2}}\left(x_{2 j}\right)
\end{aligned}
$$

and thus the measure is monotone. The proof for the upper product p-measure is analogous.

However, $\underline{\mathfrak{m}}_{12}$ and $\overline{\mathfrak{m}}_{12}$ are not, in general, product fuzzy measures of $\mu_{1}$ and $\mu_{2}$ since they can often fail to be consistent with the marginalization, i.e.

$$
\underline{\mathfrak{m}}_{12}\left(A \times X_{2}\right)=\min _{\sigma_{1}, \sigma_{2}} P_{\sigma_{1}}^{\mu_{1}} \otimes P_{\sigma_{2}}^{\mu_{2}}\left(A \times X_{2}\right)=\min _{\sigma_{1}} P_{\sigma_{1}}^{\mu_{1}}(A)
$$

that is not guaranteed to be equal to $\mu_{1}(A)$. The same happens to $\overline{\mathfrak{m}}_{12}$. However, these measures conform a bound of a product measure, as stated in the next proposition.

Proposition 6. Let $\left(X_{1}, \mathcal{A}_{X_{1}}, \mu_{1}\right)$ and $\left(X_{2}, \mathcal{A}_{X_{2}}, \mu_{2}\right)$ be measurable spaces and let $\mu_{12}^{\times}$be the $\times$-independent product of $\mu_{1}$ and $\mu_{2}$. Then, for any $H=A \times B \in \mathcal{R}$ it holds that

$$
\underline{\mathfrak{m}}_{12}(H) \leq \mu_{12}^{\times}(H) \leq \overline{\mathfrak{m}}_{12}(H)
$$

Moreover, for any $C \in \mathcal{A}_{X_{1} \times X_{2}}$,

$$
\begin{aligned}
& \underline{\mu}_{12}^{\times}(C) \leq \overline{\mathfrak{m}}_{12}(C), \\
& \underline{\mathfrak{m}}_{12}(C) \leq \bar{\mu}_{12}^{\times}(C) .
\end{aligned}
$$

Proof. According to Proposition 1,

$$
\min _{\sigma_{1}} P_{\sigma_{1}}^{\mu_{1}}(A) \leq \mu_{1}(A) \leq \max _{\sigma_{1}} P_{\sigma_{1}}^{\mu_{1}}(A)
$$

and

$$
\min _{\sigma_{2}} P_{\sigma_{2}}^{\mu_{2}}(B) \leq \mu_{2}(B) \leq \max _{\sigma_{2}} P_{\sigma_{2}}^{\mu_{2}}(B) .
$$

Multiplying both inequalities, we obtain 


$$
\begin{gathered}
\min _{\sigma_{1}} P_{\sigma_{1}}^{\mu_{1}}(A) \min _{\sigma_{2}} P_{\sigma_{2}}^{\mu_{2}}(B) \leq \mu_{1}(A) \mu_{2}(B) \leq \max _{\sigma_{1}} P_{\sigma_{1}}^{\mu_{1}}(A) \max _{\sigma_{2}} P_{\sigma_{2}}^{\mu_{2}}(B) \\
\Rightarrow \min _{\sigma_{1}, \sigma_{2}} P_{\sigma_{1}}^{\mu_{1}}(A) P_{\sigma_{2}}^{\mu_{2}}(B) \leq \mu_{1}(A) \mu_{2}(B) \leq \max _{\sigma_{1}, \sigma_{2}} P_{\sigma_{1}}^{\mu_{1}}(A) P_{\sigma_{2}}^{\mu_{2}}(B) \\
\Rightarrow \underline{\mathfrak{m}}_{12}(H) \leq \mu_{12}^{\times}(H) \leq \overline{\mathfrak{m}}_{12}(H),
\end{gathered}
$$

as $H=A \times B$ and $\mu_{12}^{\times}(H)=\mu_{1}(A) \mu_{2}(B)$. This proves Equation (31).

Now consider two sets $C \in \mathcal{A}_{X_{1} \times X_{2}}$ and $G \in \mathcal{R}$ with $G \subseteq C$. As $\overline{\mathfrak{m}}_{12}$ is a fuzzy measure, and thus monotone, it holds that $\overline{\mathfrak{m}}_{12}(G) \leq \overline{\mathfrak{m}}_{12}(C)$, which together with Equation (31) yields

$$
\mu_{12}^{\times}(G) \leq \overline{\mathfrak{m}}_{12}(G) \leq \overline{\mathfrak{m}}_{12}(C) .
$$

As Equation (33) holds for any $G \in \mathcal{R}$ with $G \subseteq C$, in particular we can write

$$
\max _{\substack{G \in \mathcal{R} \\ G \subseteq C}} \mu_{12}^{\times}(G) \leq \overline{\mathfrak{m}}_{12}(C) .
$$

Note that, according to Definition 14, the left hand side of inequality (34) is the interior product measure constructed with the product t-norm, i.e., $\underline{\mu}_{12}^{\times}(C)$, which means that $\underline{\mu}_{12}^{\times}(C) \leq \overline{\mathfrak{m}}_{12}(C)$. The remaining inequality is proven in a similar way.

The next proposition relates the concept of product p-measure with standard probabilistic product.

Proposition 7. Let $\left(X_{1}, \mathcal{A}_{X_{1}}, P_{1}\right)$ and $\left(X_{2}, \mathcal{A}_{X_{2}}, P_{2}\right)$ be probabilistic spaces. Then, $\underline{\mathfrak{m}}_{12}$ and $\overline{\mathfrak{m}}_{12}$ are equal to the standard probabilistic product.

Proof. If $P_{1}$ and $P_{2}$ are probability measures (and thus fuzzy measures after all), it follows from Definitions 2 and 3 that all the associated probability measures are the same, and therefore $\underline{\mathfrak{m}}_{12}=\overline{\mathfrak{m}}_{12}$ and they are equal to the standard probabilistic product.

The next theorem states that, for a particular class of fuzzy measures, constructing a single product measure from two marginal measures is indeed possible.

Theorem 2. Let $\left(X_{1}, \mathcal{A}_{X_{1}}, \mu_{1}\right)$ and $\left(X_{2}, \mathcal{A}_{X_{2}}, \mu_{2}\right)$ be measurable spaces such that $\mu_{1}$ and $\mu_{2}$ are monotone (alternating) capacities of order 2 , then $\underline{\mathfrak{m}}_{12}\left(\overline{\mathfrak{m}}_{12}\right)$ is a product fuzzy measure of $\mu_{1}$ and $\mu_{2}$.

Proof. According to Proposition 5, both $\underline{\mathfrak{m}}_{12}$ and $\overline{\mathfrak{m}}_{12}$ are fuzzy measures. We only have to prove that they are consistent with the marginalization, which is straightforward taking into account that, if $\mu_{1}$ and $\mu_{2}$ are capacities of order 2 , it follows from Theorem 1 that

$$
\begin{array}{ll}
\mu_{i}(A)=\min _{\sigma} P_{\sigma}^{\mu_{i}}(A), i=1,2 & \text { (for monotone capacities) } \\
\mu_{i}(A)=\max _{\sigma} P_{\sigma}^{\mu_{i}}(A), i=1,2 & \text { (for alternating capacities). }
\end{array}
$$

Thus, $\underline{\mathfrak{m}}_{12}\left(A \times X_{2}\right)=\min _{\sigma} P_{\sigma}^{\mu_{1}}(A)=\mu_{1}(A)$ and $\underline{\mathfrak{m}}_{12}\left(X_{1} \times B\right)=\min _{\sigma} P_{\sigma}^{\mu_{2}}(B)=\mu_{2}(B)$. The result for alternating capacities is analogously obtained.

Corollary 1. If $\mu_{1}$ and $\mu_{2}$ are monotone (alternating) capacities of order 2 and $A \times B \in \mathcal{R}$, then

$$
\begin{gathered}
\underline{\mathfrak{m}}_{12}(A \times B)=\mu_{1}(A) \mu_{2}(B) \quad \text { (for monotone capacities), } \\
\overline{\mathfrak{m}}_{12}(A \times B)=\mu_{1}(A) \mu_{2}(B) \quad \text { (for alternating capacities). }
\end{gathered}
$$


These results indicate that the composition of capacities of order 2 can be constructed using the product $\mathrm{p}$-measures, so that if both marginals are monotone capacities of order 2 , the product measure is $\underline{\mathfrak{m}}_{12}$, while it is $\overline{\mathfrak{m}}_{12}$ if the marginals are alternating capacities of order 2 .

\section{Functions over Product Spaces}

After studying the problem of composing fuzzy measures, the next step is to consider the construction of functions over product spaces, from functions defined on the marginal spaces.

\subsection{Composition of Functions}

Our starting point consists of two non-negative functions $h_{1}$ and $h_{2}$ defined over the reference sets $X_{1}$ and $X_{2}$, respectively. We propose the use of t-norms for carrying out the composition of the functions, and therefore we will assume, without loss of generality, that they take values on $[0,1]$.

Definition 18. Let $h_{1}$ and $h_{2}$ be functions defined on $X_{1}$ and $X_{2}$, respectively, and taking values on interval $[0,1]$. We say that $h_{12}^{\star}: X_{1} \times X_{2} \longrightarrow[0,1]$ is the $\star$-composition of $h_{1}$ and $h_{2}$ if $\forall\left(x_{1}, x_{2}\right) \in X_{1} \times X_{2}$,

$$
h_{12}^{\star}\left(x_{1}, x_{2}\right)=h_{1}\left(x_{1}\right) \star h_{2}\left(x_{2}\right),
$$

where $\star$ is a t-norm.

Example 5. Consider the functions $h_{1}$ and $h_{2}$ defined on $X_{1}$ and $X_{2}$ given in Table 2. Using the t-norm min, we find that the min-composition of $h_{1}$ and $h_{2}$, denoted as $h_{12}^{\min }$, is the function specified in Table 3.

Table 2. An example of two functions defined over the marginal spaces.

\begin{tabular}{cccc}
\hline$X_{1}$ & $h_{1}$ & $X_{2}$ & $h_{2}$ \\
\hline$x_{11}$ & 0.3 & $x_{21}$ & 0.4 \\
$x_{12}$ & 0.6 & $x_{22}$ & 0.9 \\
$x_{13}$ & 0.5 & $x_{23}$ & 0.2 \\
\hline
\end{tabular}

Table 3. Min-composition of the functions in Table 2.

\begin{tabular}{llll}
\hline & $x_{21}$ & $x_{22}$ & $x_{23}$ \\
\hline$x_{11}$ & 0.3 & 0.3 & 0.2 \\
$x_{12}$ & 0.4 & 0.6 & 0.2 \\
$x_{13}$ & 0.4 & 0.5 & 0.2 \\
\hline
\end{tabular}

Note that $h_{12}^{\star}$ is not symmetric. A possible interpretation of the composed function is the worst value of a pair $\left(x_{1 i}, x_{2 j}\right)$ regarding both marginal spaces simultaneously. For instance, a value of $h_{12}^{\star}\left(x_{12}, x_{21}\right)$ equal to 0.4 would indicate that both $h_{1}\left(x_{12}\right) \geq 0.4$ and $h_{2}\left(x_{21}\right) \geq 0.4$.

Proposition 8. Let $h_{1}$ and $h_{2}$ be functions defined on $X_{1}$ and $X_{2}$, respectively, and taking values on $[0,1]$. Then, the $\alpha$-cuts generated by $h_{12}^{\min }$, belong to the class of rectangles, $\mathcal{R}$.

Proof. The $\alpha$-cuts of any function $h$ in $X_{1} \times X_{2}$ are (see Equation (15))

$$
H_{\alpha}=\left\{\left(x_{1}, x_{2}\right) \in X_{1} \times X_{2} \mid h\left(x_{1}, x_{2}\right) \geq \alpha\right\} .
$$


Considering the min t-norm, and thus $h=h_{12}^{\min }$, we find that

$$
\begin{aligned}
H_{\alpha} & =\left\{\left(x_{1}, x_{2}\right) \in X_{1} \times X_{2} \mid h_{12}^{\min }\left(x_{1}, x_{2}\right) \geq \alpha\right\} \\
& =\left\{\left(x_{1}, x_{2}\right) \in X_{1} \times X_{2} \mid \min \left\{h_{1}\left(x_{1}\right), h\left(x_{2}\right)\right\} \geq \alpha\right\} \\
& =\left\{\left(x_{1}, x_{2}\right) \in X_{1} \times X_{2} \mid h_{1}\left(x_{1}\right) \geq \alpha \mathrm{y} h_{2}\left(x_{2}\right) \geq \alpha\right\} \\
& =\left\{x_{1} \in X_{1} \mid h_{1}\left(x_{1}\right) \geq \alpha\right\} \times\left\{x_{2} \in X_{2} \mid h_{2}\left(x_{2}\right) \geq \alpha\right\} \in \mathcal{R} .
\end{aligned}
$$

Proposition 8 guarantees that, when composing functions using the min t-norm, the resulting function generates $\alpha$-cuts belonging to class $\mathcal{R}$, which facilitates the calculation of a product measure as the interior and exterior product measures are the same in this case.

\subsection{Marginalization}

The marginalization operation acts in the opposite direction to composition, i.e., from a function defined on a product space, by applying marginalization we should obtain functions defined over the marginal spaces. In what follows, we pursue the definition of a well founded marginalization process.

Definition 19. Let $h$ be a function defined on $X_{1} \times X_{2}$ and taking values on $[0,1]$. We define the $\oplus$-marginals of has

$$
\begin{aligned}
& h_{X_{1}}^{\oplus}\left(x_{1 i}\right)=\bigoplus_{x_{2 j} \in X_{2}} h\left(x_{1 i}, x_{2 j}\right)=h\left(x_{1 i}, x_{21}\right) \oplus h\left(x_{1 i}, x_{22}\right) \oplus \ldots \oplus h\left(x_{1 i}, x_{2 m}\right), \\
& h_{X_{2}}^{\oplus}\left(x_{2 j}\right)=\bigoplus_{x_{1 i} \in X_{1}} h\left(x_{1 i}, x_{2 j}\right)=h\left(x_{11}, x_{2 j}\right) \oplus h\left(x_{12}, x_{2 j}\right) \oplus \ldots \oplus h\left(x_{1 n}, x_{2 j}\right),
\end{aligned}
$$

where $\oplus$ is a t-conorm, $n$ is the cardinality of $X_{1}$ and $m$ is the cardinality of $X_{2}$.

For instance, if we consider the max t-conorm, the marginalization process would result in

$$
h_{X_{1}}^{\max }\left(x_{1 i}\right)=\max \left\{h\left(x_{1 i}, x_{21}\right), h\left(x_{1 i}, x_{22}\right), \ldots, h\left(x_{1 i}, x_{2 m}\right)\right\} .
$$

Example 6. Consider a function $h$ defined on $X_{1} \times X_{2}$ as specified in Table 4. The corresponding marginals when the max $t$-conorm is used are shown on the last column and row. Table 5 illustrates the opposite process, where the marginals have been combined usind the min t-conorm.

Table 4. Specification of a function $h\left(x_{1 i}, x_{2 j}\right)$ and its marginals using the max t-conorm.

\begin{tabular}{ccccc}
\hline & $x_{21}$ & $x_{22}$ & $x_{23}$ & $h_{X_{1}}^{\max }$ \\
\hline$x_{11}$ & 0.5 & 0.1 & 0.2 & 0.5 \\
$x_{12}$ & 0.7 & 0.6 & 0.4 & 0.7 \\
$x_{13}$ & 0.3 & 0.5 & 0.2 & 0.5 \\
$h_{X_{2}}^{\max }$ & 0.7 & 0.6 & 0.4 & \\
\hline
\end{tabular}

Table 5. Composition of the marginals in Table 4 using the min t-conorm.

\begin{tabular}{ccccc}
\hline & $x_{21}$ & $x_{22}$ & $x_{23}$ & $h_{X_{1}}^{\max }$ \\
\hline$x_{11}$ & 0.5 & 0.5 & 0.4 & 0.5 \\
$x_{12}$ & 0.7 & 0.6 & 0.4 & 0.7 \\
$x_{13}$ & 0.5 & 0.5 & 0.4 & 0.5 \\
$h_{X_{2}}^{\max }$ & 0.7 & 0.6 & 0.4 & \\
\hline
\end{tabular}


We can notice how, in Example 6, the function obtained by composing the marginals bounds from above the original function over the product space. The next proposition shows that this property holds in rather general settings.

Proposition 9. Let $h$ be a function defined on $X_{1} \times X_{2}$ and taking values on $[0,1]$, and let $h_{X_{1}}^{\oplus}$ and $h_{X_{2}}^{\oplus}$ be the $\oplus$-marginals of $h$. Then, for any arbitrary $t$-conorm $\oplus$ it holds that

$$
h\left(x_{1 i}, x_{2 j}\right) \leq \min \left\{h_{X_{1}}^{\oplus}\left(x_{1 i}\right), h_{X_{2}}^{\oplus}\left(x_{2 j}\right)\right\}, \quad \forall\left(x_{1 i}, x_{2 j}\right) \in X_{1} \times X_{2} .
$$

Proof. According to Definition 19,

$$
h_{X_{1}}^{\oplus}\left(x_{1 i}\right)=h\left(x_{1 i}, x_{21}\right) \oplus h\left(x_{1 i}, x_{22}\right) \oplus \ldots \oplus h\left(x_{1 i}, x_{2 m}\right) .
$$

As the max t-conorm bounds any other conorm from below,

$$
h_{X_{1}}^{\oplus}\left(x_{1 i}\right) \geq \max \left\{h\left(x_{1 i}, x_{21}\right), h\left(x_{1 i}, x_{22}\right), \ldots, h\left(x_{1 i}, x_{2 m}\right)\right\}=h_{X_{1}}^{\max }\left(x_{1 i}\right) .
$$

Likewise, it holds that

$$
h_{X_{2}}^{\oplus}\left(x_{2 j}\right) \geq h_{X_{2}}^{\max }\left(x_{2 j}\right) .
$$

On the other hand, it is clear that

$$
h\left(x_{1 i}, x_{2 j}\right) \leq \min \left\{h_{X_{1}}^{\max }\left(x_{1 i}\right), h_{X_{2}}^{\max }\left(x_{2 j}\right)\right\},
$$

as $h_{X_{1}}^{\max }\left(x_{1 i}\right)$ is the maximum value of $h\left(x_{1 i}, x_{2 j}\right)$ for a fixed value $x_{1 i}$ of $X_{1}$, and $h_{X_{2}}^{\max }\left(x_{2 j}\right)$ is the maximum value of $h\left(x_{1 i}, x_{2 j}\right)$ for a fixed value $x_{2 j}$ of $X_{2}$. Therefore, combining Equation (43) with Equations (41) and (42), we obtain Equation (39).

Corollary 2. Assuming the conditions in Proposition 9, given an arbitrary t-conorm $\oplus$ it holds that, for any $\left(x_{1 i}, x_{2 j}\right) \in X_{1} \times X_{2}$

$$
h\left(x_{1 i}, x_{2 j}\right) \leq h_{12}^{\min }\left(x_{1 i}, x_{2 j}\right) .
$$

\section{Conclusions}

In this paper, we have studied the problem of constructing fuzzy measures over product domains, when fuzzy measures over the marginal spaces are available. We have proposed a definition of independence of fuzzy measures and different ways of constructing product measures that are consistent with the defined concept of independence. Even though, in general, we have only been able to give bounds for the measure on the product space when we work outside the class of rectangles $\mathcal{R}$, we show in Theorem 2 that it is possible to construct a single product measure if the marginal measures are capacities of order 2.

Our proposal for combining real functions over the marginal spaces in order to produce a joint function over the product space satisfies that the resulting function yields $\alpha$-cuts within the class of rectangles $\mathcal{R}$, if the min t-norm is used. The importance of this property is that within the class $\mathcal{R}$, we are able to compute a unique product fuzzy measure, as the interior and exterior product measures, which conform the bound of the product fuzzy measure, are the same in this case.

The results in the paper show that we are able to handle marginal spaces endowed with a fuzzy measure and a real function, and work on the product space with product measures and functions containing the information in the marginal case. Likewise, the marginal functions can be measured using, for instance, Choquet integral; the joint function can also be measured in the same way, by integrating with respect to the product measure. This provides the basic tools for defining statistical indices, as for instance indices of association, based on fuzzy measures. 
Author Contributions: Investigation, F.R., M.M. and A.S.; Writing-original draft, F.R., M.M. and A.S.; Writing-review-editing, F.R., M.M. and A.S. All authors have read and agreed to the published version of the manuscript.

Funding: This research was funded by the Spanish Ministry of Science and Innovation through grant TIN201677902-C3-3-P and by ERDF-FEDER funds.

Conflicts of Interest: The authors declare no conflicts of interest.

\section{References}

1. Choquet, G. Theory of capacities. Ann. L'Institut Fourier 1954, 5, 131-295. [CrossRef]

2. Li, J. On Null-Continuity of Monotone Measures. Mathematics 2020, 8, 205. [CrossRef]

3. Sugeno, M. Theory of Fuzzy Integrals and Its Applications. Ph.D. Thesis, Tokyo Institute of Technology, Tokyo, Japan, 1974.

4. Dempster, A.P. Upper and lower probabilities induced by a multivalued mapping. Ann. Math. Stat. 1967, 38, 325-339. [CrossRef]

5. Shafer, G. A Mathematical Theory of Evidence; Princeton University Press: Princeton, NJ, USA, 1976.

6. Nedović, L.; Pap, E. Aggregation of sequence of fuzzy measures. Iran. J. Fuzzy Syst. 2020, 17, 39-55.

7. Narukawa, Y.; Torra, V. On distorted probabilities and $m$-separable fuzzy measures. Int. J. Approx. Reason. 2011, 52, 1325-1336. [CrossRef]

8. Murillo, J.; Guillaume, S.; Bulacio, P. k-maxitive fuzzy measures: A scalable approach to model interactions. Fuzzy Sets Syst. 2017, 324, 33-48. [CrossRef]

9. Combarro, E.; Miranda, P. On the structure of the $k$-additive fuzzy measures. Fuzzy Sets Syst. 2010, 161, 2314-2327. [CrossRef]

10. Navarro, J.; Spizzichino, F. Aggregation and signature based comparisons of multi-state systems via decompositions of fuzzy measures. Fuzzy Sets Syst. 2020, 396, 115-137. [CrossRef]

11. Denneberg, D. Conditioning (updating) non-additive measure. Ann. Oper. Res. 1994, 52, 21-42. [CrossRef]

12. Denneberg, D. Non-Additive Measure and Integral; Theory and Decision Library B; Springer: Dordrecht, Netherlands, 1994.

13. Hendon, E.; Jacobsen, H.J.; Sloth, B.; Tranæs, T. The product of capacities and belief functions. Math. Soc. Sci. 1996, 32, 95-108. [CrossRef]

14. Ghirardato, P. On idependence for Non-Additive measures, with Fubini theorem. J. Econ. Theory 1997, 73, 261-291. [CrossRef]

15. Koshevoy, G.A. Distributive lattice and product of capacities. J. Math. Anal. Appl. 1998, 219, 427-441. [CrossRef]

16. Denneberg, D. Totally monotone core and products of monotone measures. Int. J. Approx. Reason. 2000, 24, 273-281. [CrossRef]

17. Denneberg, D. Conditional expectation for monotone measure, the discrete case. J. Math. Econ. 2002, 37, $105-121$. [CrossRef]

18. Brüning, M.; Denneberg, D. Max-min $\sigma$-additive representation of monotone measure. Stat. Pap. 2002, 43, 23-35. [CrossRef]

19. Candeloro, D.; Mesiar, R.; Sambucini, A. A special class of fuzzy measures: Choquet integral and applications. Fuzzy Sets Syst. 2019, 355, 83-99. [CrossRef]

20. Beliakov, G.; James, S.; Wu, J. Discrete Fuzzy Measures. In Studies in Fuzziness and Soft Computing; Springer International Publishing: Cham, Switzerland, 2020; Volume 382.

21. De Campos, L.M.; Bolaños, M.J. Representation of fuzzy measures through probabilities. Fuzzy Sets Syst. 1989, 31, 23-36. [CrossRef]

22. De Campos, L.M. Caracterización y Estudio de Medidas Difusas a Partir de Probabilidades. Ph.D. Thesis, Universidad de Granada, Granada, Spain, 1987.

23. Jia, G.; Zhang, N. New proofs for several properties of capacities. arXiv 2013, arXiv:1307.0913v1.

24. Schweizer, B.; Sklar, A. Probability Metric Spaces; Elsevier: North-Holland, NY, USA, 1983.

25. Zadeh, L.A. Fuzzy sets. Inf. Control 1965, 8, 338-353. [CrossRef]

26. Dubois, D.; Prade, H. Fuzzy Sets and Systems: Theory and Applications. In Mathematics in Science and Engineering; Academic Press: Cambridge, MA, USA, 1980; Volume 114. 
27. Grabisch, M.; Nguyen, H.T.; Walker, E.A. Fundamentals of Uncertainty Calculi with Applications to Fuzzy Inference; Theory and Decision Library B; Springer: Dordrecht, The Netherlands, 1995.

28. Klement, E.P. Some mathematical aspects of fuzzy sets: Triangular norms, fuzzy logic, and generalized measures. Fuzzy Sets Syst. 1997, 90, 133-140. [CrossRef]

29. Grabisch, M.; Murofushi, T.; Sugeno, M., Eds. Fuzzy Measure and Integrals. In Studies in Fuzziness and Soft Computing; Physica Verlag: Heidelberg, Germany, 2000; Volume 40.

30. Bauer, H. Probability Theory and Elements of Measure Theory, 2nd ed.; Academic Press: London, UK, 1981.

31. De Cooman, G. Possibility theory I: The measure and integral theoretic groundwork. Int. J. Gen. Syst. 1997, 25, 291-323. [CrossRef]

32. De Cooman, G.; Zhang, G.; Kerre, E. Possibility measures and possibility integrals defined on a complete lattice. Fuzzy Sets Syst. 2001, 120, 459-467. [CrossRef]

(C) 2020 by the authors. Licensee MDPI, Basel, Switzerland. This article is an open access article distributed under the terms and conditions of the Creative Commons Attribution (CC BY) license (http://creativecommons.org/licenses/by/4.0/). 